\title{
Ferroelectric hexagonal and rhombic monolayer ice phases $\dagger$
}

\begin{abstract}
Wen-Hui Zhao, ${ }^{a}$ Jaeil Bai, ${ }^{b}$ Lan-Feng Yuan, ${ }^{* a}$ Jinlong Yang ${ }^{a}$ and Xiao Cheng Zeng ${ }^{* b}$
Two new phases of water, the mid-density hexagonal monolayer ice and the high-density flat rhombic monolayer ice, are observed in our molecular dynamics simulations of monolayer water confined between two smooth hydrophobic walls. These are in addition to the two monolayer ices reported previously, namely, the low-density $4 \cdot 8^{2}$ monolayer ice and the high-density puckered rhombic monolayer ice (HD-pRMI). Stabilities of the structures are confirmed by ab initio computation. Importantly, both new phases and the HD-pRMI are predicted to be ferroelectric. An in-plane external electric field can further stabilize these ferroelectric monolayer ices.
\end{abstract}

Received 9th December 2013 Accepted 6th January 2014

DOI: $10.1039 / c 3 s c 53368 a$

www.rsc.org/chemicalscience polymorphs have been predicted via simulations or observed in experiments, including trilayer hexagonal and rhombic ices, ${ }^{\mathbf{1 6}}$ bilayer hexagonal, rhombic, amorphous, very-high-density amorphous, and quasi-crystalline ices, ${ }^{17-24}$ and the monolayer Archimedean $4 \cdot 8^{2}$ truncated square tiling, ${ }^{25,26}$ rhombic $^{26-29}$ and flat hexagonal ${ }^{30}$ ices. However, regarding ferroelectricity in $2 \mathrm{D}$ ices, the most relevant report is a "probably ferroelectric" monolayer ice formed epitaxially on mica and observed via atomic force microscopy (AFM).$^{31}$ To date, a definite report of ferroelectric $2 \mathrm{D}$ ice is still lacking.

In this paper, we report two new monolayer ices via MD simulations, namely, a mid-density hexagonal monolayer ice (MD-HMI) and a high-density flat rhombic monolayer ice (HD-fRMI). Also studied are two known monolayer ices, namely, the low-density $4 \cdot 8^{2}$ monolayer ice (LD-48MI ${ }^{26}$ and the HD-pRMI. ${ }^{26-29}$ The three phases of MD-HMI, HD-fRMI and HD-pRMI are observed to show spontaneous polarization and ferroelectric hysteresis, therefore, we validate them as $2 \mathrm{D}$ ferroelectric ices.

\section{Methods}

The simulation system consists of 400 water molecules confined between two hydrophobic planar walls. The wall separation $D$ is set within the range of 0.51 to $0.65 \mathrm{~nm}$, in which only one layer of water can be accommodated. The TIP5P potential ${ }^{32}$ is used to describe water-water interaction, as used previously by Zangi and Mark ${ }^{\mathbf{1 6}}$ to simulate electrofreezing of a water slab under an in-plane electric field ( $5 \mathrm{~V} \mathrm{~nm}^{-1}$ ) and by Qiu and $\mathrm{Guo}^{33}$ to study the electromelting of confined monolayer ice. The water-wall interactions are described by the Lennard-Jones (L-J) 9-3 potential function with the parameters $\sigma_{\mathrm{o}-\mathrm{wl}}=0.25 \mathrm{~nm}$ and $\varepsilon_{\mathrm{o}-\mathrm{wl}}$ $=1.25 \mathrm{~kJ} \mathrm{~mol}^{-1}$ (following ref. 20 and 28), namely, the integral of the L-J 12-6 potential for a structureless wall. This is because our previous study demonstrates that the formation of LD-48MI 
is insensitive to the atomic structure of the wall surface by using two structureless walls and two single graphene layers, ${ }^{26}$ and our new MD simulations reinforce this point (see Fig. $\mathrm{S} 1 \dagger$ in ESI for detail). All the MD simulations are performed in the constant lateral pressure $\left(P_{\mathrm{L}}\right)$ and constant temperature $(T)$ ensemble with periodic boundary conditions in the lateral directions ( $x$ and $y$ ), using the GROMACS 4.5 package. ${ }^{34}$ Temperature and pressure are controlled by the Nosé-Hoover thermostat $^{35}$ and Parrinello-Rahman barostat, ${ }^{36}$ respectively. A cutoff of $1 \mathrm{~nm}$ is used for the $\mathrm{LJ}$ interactions, and the long-range electrostatic interactions are treated by the slab-adapted Ewald sum method. ${ }^{37}$

\section{Results and discussion}

First, we performed molecular dynamics simulations for a given $D=0.557 \mathrm{~nm}$, a value used in previous molecular dynamics simulations of monolayer water but with a switching function to cut off all interaction at $0.875 \mathrm{~nm} .^{26,29}$ The confined water was cooled from $320 \mathrm{~K}$ to $210 \mathrm{~K}$ at $P_{\mathrm{L}}=1 \mathrm{MPa}$ in a step of $10 \mathrm{~K}$. At each temperature, the simulation lasted $20 \mathrm{~ns}$. Then the system was equilibrated at $200 \mathrm{~K}$ and $P_{\mathrm{L}}=-50 \mathrm{MPa}$ for $60 \mathrm{~ns}$. A monolayer crystalline structure consisting of tetragons and octagons (with some voids) formed spontaneously, which is LD48MI (with some voids). Fig. 1a displays its inherent structure (obtained by applying the steepest-descent method to the final configuration of the system ${ }^{18}$ ).

Taking the LD-48MI at $D=0.557 \mathrm{~nm}$ as the initial configuration, thirteen additional independent simulations were performed for $D=0.51,0.52,0.53,0.54,0.55,0.57,0.58,0.59,0.6$, $0.62,0.63,0.64$, and $0.65 \mathrm{~nm}$, all at $P_{\mathrm{L}}=-50 \mathrm{MPa}$ and $T=200 \mathrm{~K}$. The LD-48MI structure is still intact in most cases except for at $D$ $=0.64$ and $0.65 \mathrm{~nm}$, at which the LD-48MI melts into a monolayer liquid (MLiq). Next, for all fourteen $D$ values, we compressed isothermally the monolayer water stepwise from -50 to $800 \mathrm{MPa}$, scanning through 11-14 state points of $P_{\mathrm{L}}$ (20 ns for each state point). In these sequential simulations, three other monolayer crystalline phases were attained,
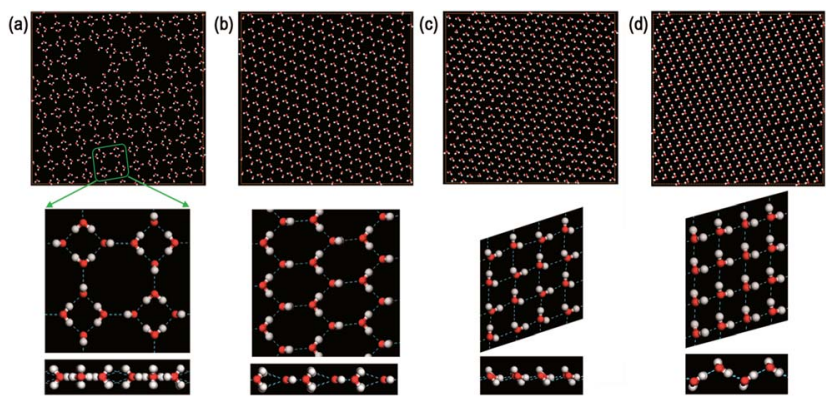

Fig. 1 The inherent structures of 2D monolayer ices formed between two hydrophobic walls at $T=200 \mathrm{~K}$. (a) LD-48MI (at $D=0.557 \mathrm{~nm}$ and $P_{\mathrm{L}}=-50 \mathrm{MPa}$ ). (b) MD-HMI (at $D=0.557 \mathrm{~nm}$ and $P_{\mathrm{L}}=100 \mathrm{MPa}$ ). (c) HD-fRMI (at $D=0.52 \mathrm{~nm}$ and $P_{\mathrm{L}}=600 \mathrm{MPa}$ ). (d) HD-pRMI (at $D=$ $0.6 \mathrm{~nm}$ and $P_{\mathrm{L}}=600 \mathrm{MPa}$ ). Upper panels: top view. Middle panels: zoomed top view. Lower panels: zoomed side view. Red and white spheres represent oxygen and hydrogen atoms, and blue dotted lines hydrogen bonds. including the two new structures MD-HMI (Fig. 1b) and HDfRMI (Fig. 1c), and the known structure HD-pRMI (Fig. 1d). Structural features and properties of these monolayer ices are discussed below.

Fig. 2 shows the area density $\rho$ of monolayer water as $P_{\mathrm{L}}$ increases for various given $D$ at $200 \mathrm{~K}$. Based on the number of phase transitions in every isotherm, the change in $\rho$ and the corresponding phase behavior can be classified into three types for three ranges of $D$ : (1) for $0.51 \mathrm{~nm} \leq D \leq 0.58 \mathrm{~nm}$, three phase transitions take place in sequence. As $P_{\mathrm{L}}$ increases from -50 MPa to 50-100 MPa, the LD-48MI transforms into the middensity hexagonal monolayer ice, i.e. MD-HMI (Fig. 1b). The transition from LD-48MI to MD-HMI exhibits the Oswald staging phenomenon, namely, an unstable intermediate liquid state during the solid-to-solid transition (Movie S1†). Interestingly, a stable monolayer liquid (MLiq) can also be observed by increasing the $P_{\mathrm{L}}$ to $300-400 \mathrm{MPa}$. The lateral diffusion constant of MLiq is $D_{\mathrm{L}} \approx 6 \times 10^{-6} \mathrm{~cm}^{2} \mathrm{~s}^{-1}$, which is about $30 \%$ of the diffusion constant of bulk water under ambient conditions $\left(2.2 \times 10^{-5} \mathrm{~cm}^{2} \mathrm{~s}^{-1}\right)$, but much greater than those of the four monolayer solids (LD-48MI, $D_{\mathrm{L}} \approx 1 \times 10^{-8} \mathrm{~cm}^{2} \mathrm{~s}^{-1}$; MD-HMI, $D_{\mathrm{L}} \approx 7 \times 10^{-8} \mathrm{~cm}^{2} \mathrm{~s}^{-1}$; HD-fRMI, $D_{\mathrm{L}} \approx 1 \times 10^{-7} \mathrm{~cm}^{2} \mathrm{~s}^{-1}$; and HD-pRMI, $D_{\mathrm{L}} \approx 1 \times 10^{-9} \mathrm{~cm}^{2} \mathrm{~s}^{-1}$ ). As $P_{\mathrm{L}}$ reaches $500-600 \mathrm{MPa}$, the MLiq freezes into one of the two rhombic structures: either HD-fRMI (Fig. 1c) for $0.51 \mathrm{~nm} \leq D \leq 0.57 \mathrm{~nm}$ or HD-pRMI (Fig. 1d) for $D=0.58 \mathrm{~nm}$. Also, importantly, the pressure range in which MD-HMI is stable shrinks with the increase of $D$. For $D=0.58 \mathrm{~nm}$, MD-HMI appears only in the pressure range of $60 \mathrm{MPa} \leq P_{\mathrm{L}} \leq 90 \mathrm{MPa}$ (we examine more pressure states for this case). In a wider nanoslit ( $D \geq 0.59 \mathrm{~nm})$, the MD-HMI is no longer stable.

(2) For $0.59 \mathrm{~nm} \leq D \leq 0.63 \mathrm{~nm}$, two phase transitions take place in sequence as $P_{\mathrm{L}}$ increases, namely, from LD-48MI to MLiq and then to HD-pRMI. Compared to (1), the MD-HMI phase is bypassed. (3) For $0.64 \mathrm{~nm} \leq D \leq 0.65 \mathrm{~nm}$, only one phase transition takes place as $P_{\mathrm{L}}$ increases. Here, the state below $P_{\mathrm{L}}=-50 \mathrm{MPa}$ is MLiq rather than LD-48MI. As $P_{\mathrm{L}}$ increases, the MLiq crystallizes into HD-pRMI.

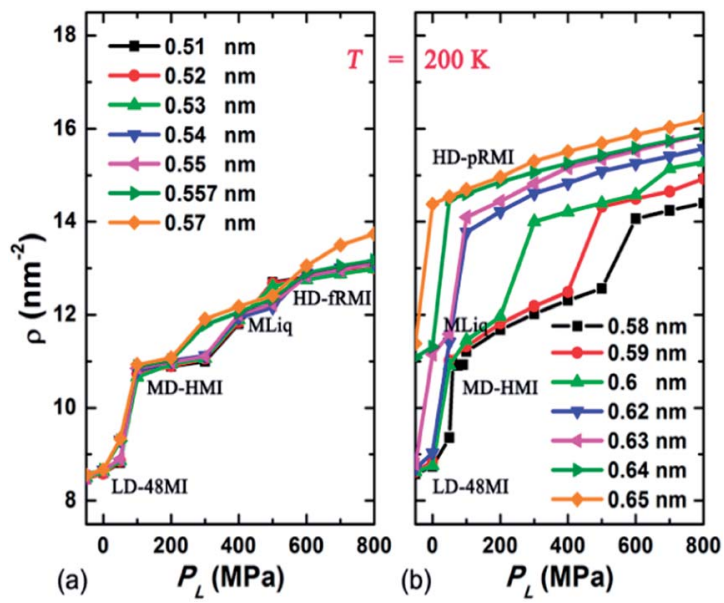

Fig. 2 The area density $\rho$ of monolayer water versus the lateral pressure $P_{\mathrm{L}}$ for various given $D$ at $200 \mathrm{~K}$. 
An approximate $P_{\mathrm{L}}-D$ phase diagram of monolayer water (under zero external electric field) is plotted based on results of the MD simulations (Fig. 3a). We use the two-phase coexistence simulation method with the $N P_{\mathrm{L}} T$ ensemble to determine the phase boundaries (see ESI $\dagger$ ). More accurate phase boundaries can be achieved via the multibaric-multithermal ensemble approach that involves an anisotropic pressure control. ${ }^{38} \mathrm{~A}$ conspicuous feature is that a MLiq domain fully separates two solid phases. On the left side of the MLiq domain, the MD-HMI phase arises in the higher $P_{\mathrm{L}}$ region; while on the right side of the MLiq domain, the HD-pRMI phase arises in the larger $D$ region. There exist two triple points: one for LD-48MI, MLiq, and MD-HMI, and the other for HD-fRMI, MLiq, and HD-pRMI.

In the inherent structure of LD-48MI (Fig. 1a), it can be seen that the molecular plane of each water molecule is perpendicular to the molecular plane of its nearest-neighbor molecules. Each water molecule has three nearest-neighbors, but entails four hydrogen bonds; namely, LD-48MI satisfies the ice rule. This is because the molecule whose molecular plane is perpendicular to the two walls acts as a "double donor to a single acceptor", i.e., its two $\mathrm{O}-\mathrm{H}$ arms are obliquely oriented towards one $\mathrm{O}$ atom in its nearest-neighbor in-plane water molecule, forming two weak hydrogen bonds. Meanwhile, as a "double acceptor of double donors", this molecule forms two strong hydrogen bonds with its two other nearest-neighbor molecules. An in-plane water molecule entails two strong and two weak hydrogen bonds as well, but as a "double donor to double acceptors" and a "double acceptor of a single donor". In
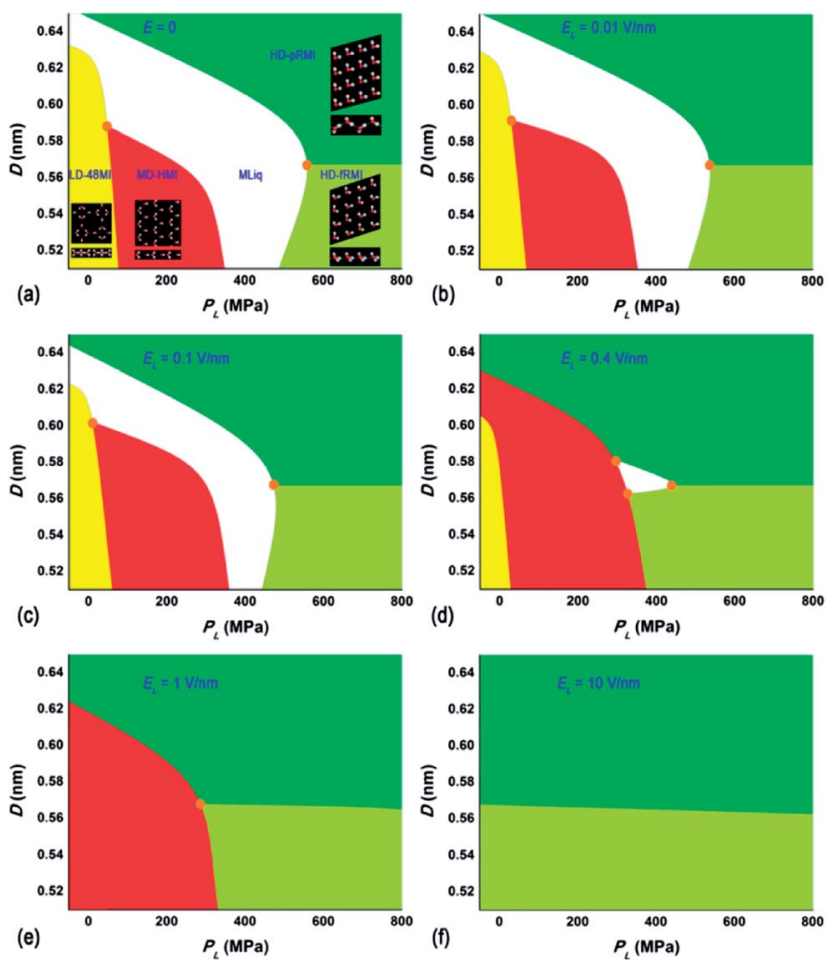

Fig. 3 Semi-quantitative phase diagrams of monolayer water confined between two hydrophobic walls at $200 \mathrm{~K}$ with the lateral electric field strength $E_{\mathrm{L}}=$ (a) 0 , (b) 0.01 , (c) 0.1 , (d) 0.4 , (e) 1.0 , and (f) 10 $\mathrm{V} \mathrm{nm}{ }^{-1}$, respectively. Triple points are highlighted by orange circles. a plot of the distribution of $\mathrm{O}-\mathrm{H} \cdots \mathrm{O}$ angles of hydrogen bonds (Fig. 4a), the two peaks around $164^{\circ}$ and $115^{\circ}$ (for $D \leq 0.55 \mathrm{~nm}$ ) correspond to the strong and weak hydrogen bonds, respectively. With the increase of $D$, the libration of the two $\mathrm{O}-\mathrm{H}$ bonds involved in the weak hydrogen bonds with the same $\mathrm{O}$ atom becomes stronger. Thus, the peak around $115^{\circ}$ is lowered and gradually becomes a shoulder peak.

The density distribution of oxygen atoms in the $z$ direction (normal to the walls), i.e. the transverse density profile of LD$48 \mathrm{MI}\left(P_{\mathrm{L}}=-50 \mathrm{MPa}\right.$, for $\left.0.51 \mathrm{~nm} \leq D \leq 0.63 \mathrm{~nm}\right)$, is unimodal, as shown in Fig. 5a. As a measure of the planarity of monolayer ices, the width of the unimodal peak is less than $0.05 \mathrm{~nm}$. This width increases with $D$, and as $D$ reaches $0.64 \mathrm{~nm}$, the distribution becomes bimodal, corresponding to the MLiq. As shown by the lateral oxygen-oxygen radial distribution function (RDF) $g^{\mathrm{oo}}\left(r_{x y}\right)$ in Fig. 6, the nearest neighbor O-O distance in LD-48MI is $0.28 \mathrm{~nm}$, close to the nearest neighbor distance for the bulk water. The second peak is at $0.39 \mathrm{~nm}$, corresponding to the diagonal distance of a tetragon $(\sqrt{ } 2 \times 0.28 \mathrm{~nm})$. The third peak is at $0.51 \mathrm{~nm}$, corresponding to the distance between a molecule and its second nearest-neighbor in an octagon $\left(2 \times \sin \left(135^{\circ} / 2\right)\right.$ $\times 0.28 \mathrm{~nm}$ ). Because each molecule has a para-position in a tetragon and two meta-positions in an octagon, the height of the third peak is about twice of that of the second peak. The position and height of the following peaks can be also understood based on the Archimedean $4 \cdot 8^{2}$ truncated square tiling.

Second, we focus on the new monolayer ice structure MDHMI. Like LD-48MI, every water molecule in MD-HMI also has three nearest-neighbors, and the plane of each water molecule is also perpendicular to those of its three nearest-neighbor molecules (Fig. 1b). Interestingly, the hexagonal framework still meets the ice rule in the same fashion as LD-48MI, i.e., every molecule acts as either a "double donor to a single acceptor" or as a "double acceptor of a single donor", forming two weak and two strong hydrogen bonds (with $\mathrm{O}-\mathrm{H} \cdots \mathrm{O}$ angles around $106^{\circ}$ and $164^{\circ}$ as shown in Fig. $4 \mathrm{~b}$ ).

Despite many similarities in the hydrogen bonding networks of MD-HMI and LD-48MI, there is a fundamental distinction on their global polarizations. In LD-48MI, the dipole moments of water molecules offset one another, resulting in zero total dipole. On the other hand, the dipole vectors of all the molecules in MD-HMI are all parallel to the longest diagonal of a hexagon, i.e., MD-HMI exhibits spontaneous polarization. For $D=0.52 \mathrm{~nm}$, its polarization is $15 \mu \mathrm{C} \mathrm{cm}^{-2}$. A strong hysteresis loop for the MD-HMI is also observed from additional MD simulations, confirming the MD-HMI as being ferroelectric (Fig. 7a).

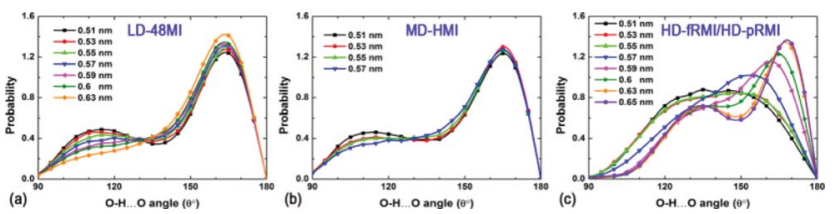

Fig. 4 Hydrogen-bond angle distributions at $T=200 \mathrm{~K}$ for (a) LD$48 \mathrm{Ml}$ at $P_{\mathrm{L}}=-50 \mathrm{MPa}$, (b) MD-HMI at $P_{\mathrm{L}}=100 \mathrm{MPa}$, and (c) HD-fRMI and $\mathrm{HD}$-pRMI at $P_{\mathrm{L}}=800 \mathrm{MPa}$, respectively. 

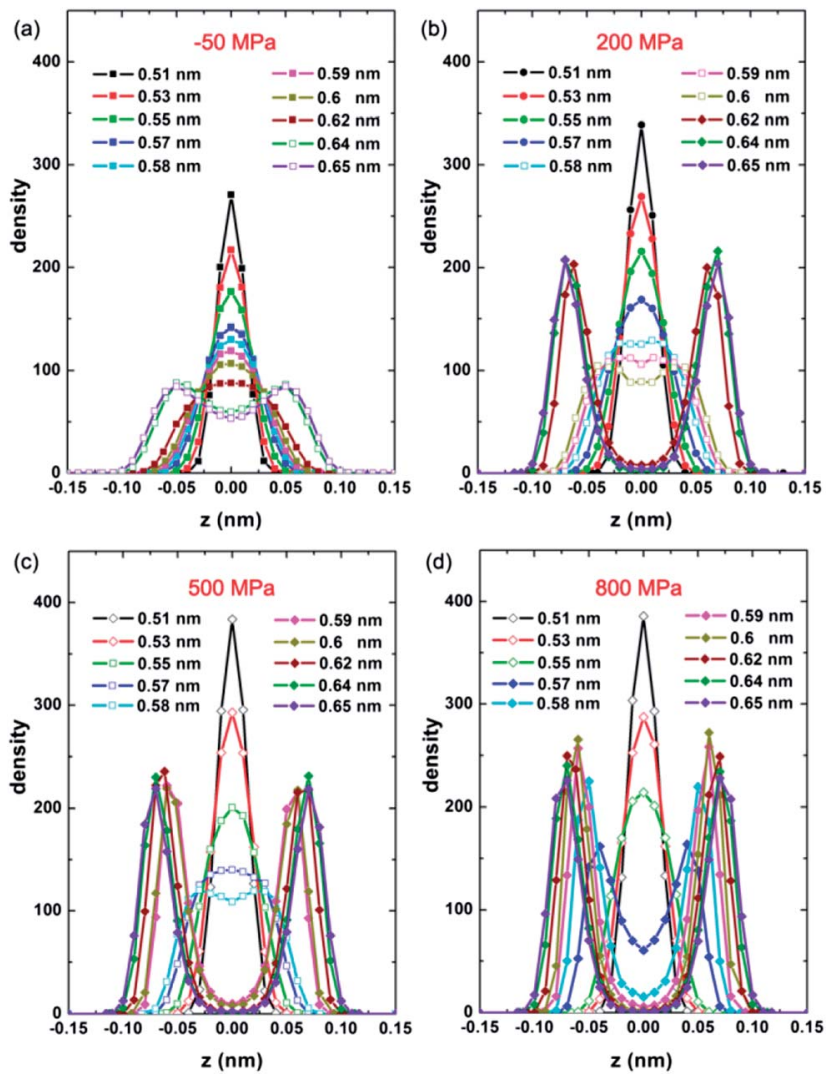

Fig. 5 The transverse density profile for different slit width $D$ at $P_{\mathrm{L}}=(\mathrm{a})$ $-50 \mathrm{MPa}$, (b) $200 \mathrm{MPa}$, (c) $500 \mathrm{MPa}$, and (d) $800 \mathrm{MPa}$, respectively. The filled squares indicate LD-48MI, unfilled squares MLiq, filled circles MD-HMI, unfilled diamonds HD-fRMI, and filled diamonds HD-pRMI.

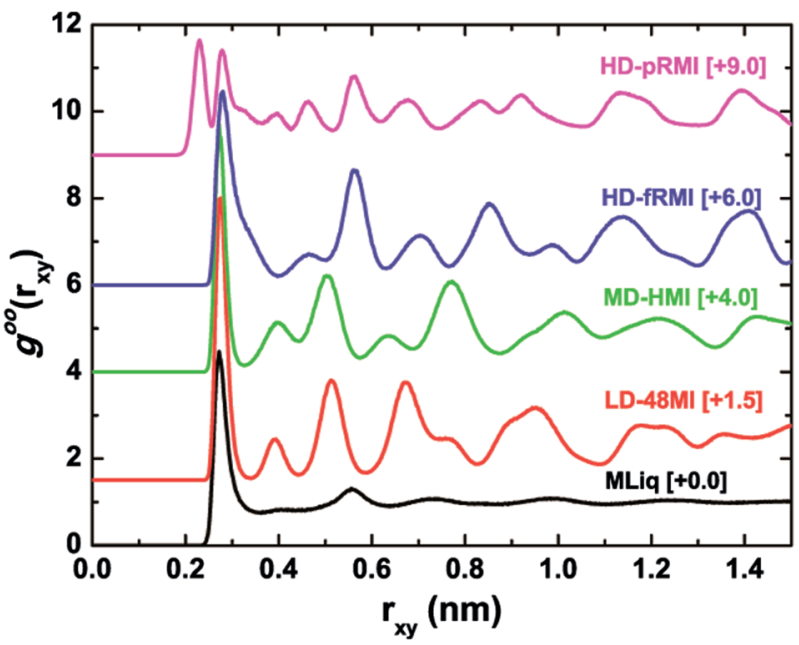

Fig. 6 Lateral oxygen-oxygen radial distribution functions $g^{\circ \circ}\left(r_{x y}\right)$ at $T$ $=200 \mathrm{~K}$ for MLiq $\left(P_{\mathrm{L}}=400 \mathrm{MPa}, D=0.557 \mathrm{~nm}\right)$, LD-48MI $\left(P_{\mathrm{L}}=\right.$ $-50 \mathrm{MPa}, D=0.557 \mathrm{~nm}), \mathrm{MD}-\mathrm{HMI}\left(P_{\mathrm{L}}=100 \mathrm{MPa}, D=0.557 \mathrm{~nm}\right), \mathrm{HD}-$ fRMI $\left(P_{\mathrm{L}}=600 \mathrm{MPa}, D=0.52 \mathrm{~nm}\right)$, and HD-pRMI $\left(P_{\mathrm{L}}=600 \mathrm{MPa}, D=\right.$ $0.64 \mathrm{~nm})$.

We also performed $a b$ initio computation using the CASTEP program $^{39}$ to test the stabilities and polarization of the monolayer ices (see ESI $\dagger$ for more detail). As shown in Fig. 8, a

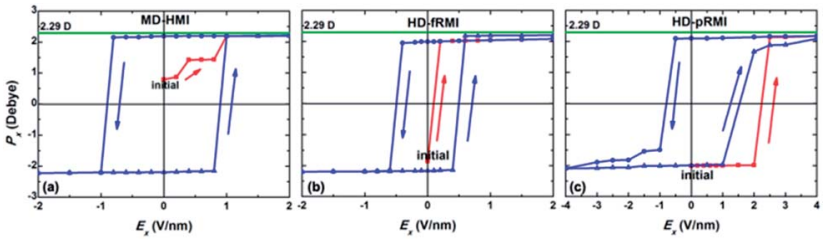

Fig. 7 Hysteresis loops of polarization, $P_{x}$, per water molecule for MD$\mathrm{HMI}, \mathrm{HD}$-fRMI, and HD-pRMI. The electric field $E$ was applied along the $x$ axis. The closed squares are the initial polarization. The green line $(2.29 \mathrm{D})$ denotes the permanent electric dipole moment of TIP5P water. $^{32}$

monolayer ice confined between two graphene monolayers with a separation of $D^{\prime}=0.47 \mathrm{~nm}$ is identical to the MD-HMI, whose polarization is $20 \mu \mathrm{C} \mathrm{cm}^{-2}$, whereas a free-standing LD-48MI is stable with the same structural features as observed from our classical MD simulations, and its polarization is 0 .

As Fig. 5b shows, the transverse density profile of the MDHMI (at $P_{\mathrm{L}}=200 \mathrm{MPa}$ ) is also characterized by a Gaussian-like distribution with a width $<0.05 \mathrm{~nm}$, and this width also increases with $D$. For $D=0.58 \mathrm{~nm}$, the MD-HMI melts into MLiq, and the density distribution starts to develop two peaks, while the value of the density profile at $z=0$ (the central plane parallel to the two walls) is still significant. For $D=0.62 \mathrm{~nm}$, the value at $z=0$ drops to almost zero, reflecting a phase transition from MLiq to HD-pRMI. The first peak in the lateral O-O RDF of the MD-HMI is at $\sim 0.27 \mathrm{~nm}$ (Fig. 6), a value again close to that of the nearest-neighbor distance in bulk water. (a)

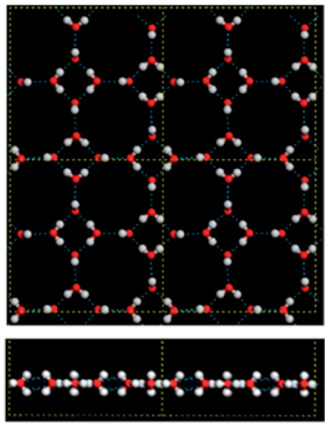

(c)

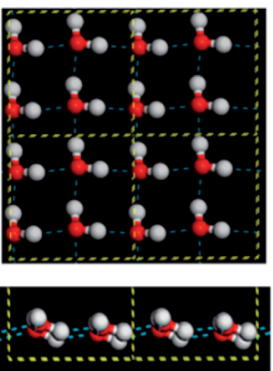

(b)

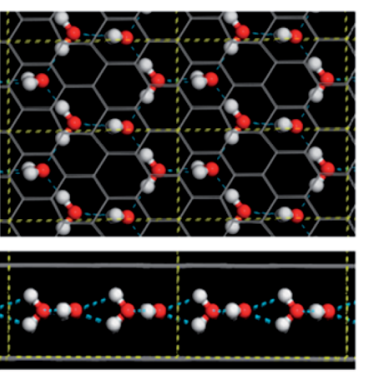

(d)

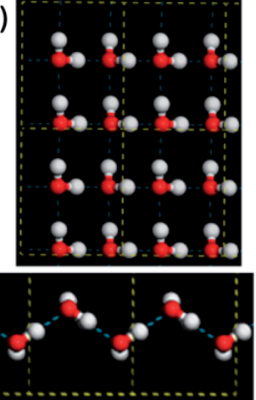

Fig. 8 The optimized structures of monolayer ices (upper panel: top view; lower panel: side view) (a) LD-48MI $\left(L_{x}=13.46 \AA, L_{y}=13.46 \AA\right)$, (b) MD-HMI confined between two graphene layers $\left(L_{x}=8.67 \AA, L_{y}=\right.$ $4.62 \AA)$, (c) HD-fRMI ( $\left.L_{x}=5.52 \AA, L_{y}=5.5 \AA\right)$, and (d) HD-pRMI $\left(L_{x}=\right.$ $\left.5.62 \AA, L_{y}=4.62 \AA\right)$. $L_{x}$ and $L_{y}$ are lattice constants of the supercell. Yellow dashed lines illustrate the boundary of the supercell. Blue dashed lines represent hydrogen bonds among water molecules. Gray lines in (b) represent the graphene layers. 
Third, we turn our attention to the two high-density monolayer ices HD-fRMI and HD-pRMI. As shown in Fig. 1c and d, both HD-fRMI and HD-pRMI are composed of rhombic rings. The primary structural distinction between them can be seen from the side view shown in Fig. 1c and d: the oxygen atoms of the HD-fRMI are essentially in the same plane parallel to the walls, while those of the HD-pRMI are in alternate ridges with different $z$ coordinates. As shown in Fig. $5 c$ and d, the transverse density profile of the HD-fRMI is unimodal, while that of the HD-pRMI is bimodal, which is why we name this ice as "puckered". Still, one may wonder whether both ice structures belong to the same phase. To address this question, we carried out an additional 32 independent molecular dynamics simulations at $P_{\mathrm{L}}=800 \mathrm{MPa}$ and $T=200 \mathrm{~K}$ for increasing $D$ stepwise from $0.55 \mathrm{~nm}$ (HD-fRMI) to $0.58 \mathrm{~nm}$ (HD-pRMI) and then for the reverse (decreasing $D$ ) process. As shown in Fig. 9, when $D$ increases, the potential energy $U$ first slowly decreases and then drops steeply by about $1.5 \mathrm{~kJ} \mathrm{~mol}^{-1}$ for $D$ from $0.570 \mathrm{~nm}$ to $0.574 \mathrm{~nm}$. Meanwhile, the area density $\rho$ first increases gradually, and then jumps up by about $0.8 \mathrm{~nm}^{-2}$. Upon decreasing $D$, $U$ and $\rho$ also show abrupt changes in the reverse process, but with a clear hysteresis. So we conclude that the HD-fRMI is a new phase different from the HD-pRMI, and the transition between the two phases is first-order upon changing $D$.

Why is such a transition first order? To answer the question, we analyze the hydrogen-bonding networks of the two highdensity ice phases. Note that the coordination number of every water molecule in the HD-fRMI and HD-pRMI is four, as opposed to three in the LD-48MI and MD-HMI. As such, it seems that water molecules can meet the ice rule more easily without invoking the "double donor to a single acceptor" mechanism. Nevertheless, another issue arises: the $\mathrm{O}-\mathrm{H} \cdots \mathrm{O}$ angles of the hydrogen bonds, i.e., where the $\mathrm{H}$ atoms between the two $\mathrm{O}$ atoms of neighboring molecules are located. Normally, the strength of a hydrogen bond increases with its $\mathrm{O}-\mathrm{H} \cdots$ $O$ angle, and a linear hydrogen bond would be the most ideal. The HD-fRMI can make a stable hydrogen-bonding network by arranging all atoms of every water molecule in the same plane and the two $\mathrm{H}$ atoms of each molecule roughly in two lines connecting with its own $\mathrm{O}$ atom and with its two neighbor $\mathrm{O}$ atoms, respectively (we call this arrangement as "all-atom planar structure"). As the $\mathrm{H}-\mathrm{O}-\mathrm{H}$ bond angle of water molecules $\left(104.5^{\circ}\right)$ is close to the inner rhombus angle of HD-fRMI $\left(108^{\circ}\right)$, all the $\mathrm{O}-\mathrm{H} \cdots \mathrm{O}$ angles would be close to $180^{\circ}$. However, analysis
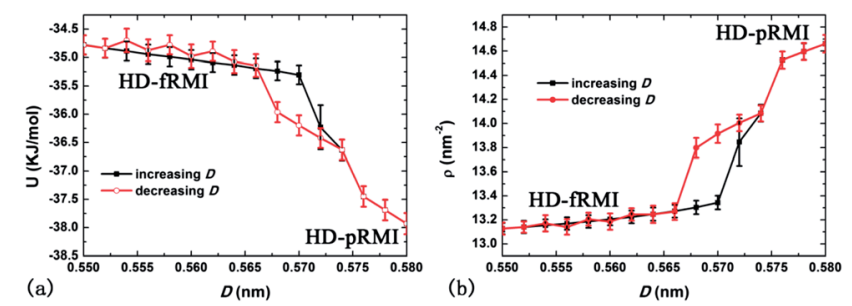

Fig. 9 The potential energy $U$ (a) and area density $\rho$ (b) at $P_{\mathrm{L}}=$ $800 \mathrm{MPa}$ and $T=200 \mathrm{~K}$ with increasing $D$ first, followed by the decreasing $D$ process. of the electronic structure indicates that this all-atom planar arrangement is unfavorable. The $\mathrm{O}$ atom in water molecule is $\mathrm{sp}^{3}$ hybridized, and its four molecular-orbital lobes yield two covalent bonds and two lone electron pairs. When forming hydrogen bonds, the two lone pairs of the $\mathrm{O}$ atom act as $\mathrm{H}$ acceptor. As such, the two $\mathrm{O}-\mathrm{H}$ covalent bonds and associated two $\mathrm{O} \cdots \mathrm{H}$ vectors of the hydrogen bonds tend to point to the four vertexes of a tetrahedron centered from the $\mathrm{O}$ atom. If the hydrogen bonds are in the same plane as the covalent bonds, they would cause too large an energy penalty. Our ab initio density-functional calculations indeed show that the all-atom planar structure is unstable.

An ideal hydrogen-bonding network of water should satisfy the three requirements simultaneously: (1) four hydrogen bonds for every water molecule, (2) tetrahedral orientation for the four hydrogen bonds, as well as (3) nearly linear hydrogen bonds. Ice structures with the coordination number of three cannot meet the first and the third requirement at the same time. Hence, both LD-48MI and MD-HMI favor the first and the second requirements while compromise on the third requirement, resulting in two nearly linear and two bent hydrogen bonds for each water molecule.

In the HD-fRMI, the geometric constraints to meet the three requirements are even stronger. Suppose there are three $\mathrm{O}$ atoms in a linear row $(\mathrm{O} 0, \mathrm{O} 1$, and $\mathrm{O} 2$ where $\mathrm{O} 1$ is between $\mathrm{O} 0$ and $\mathrm{O} 2$ ) with distance being $\sim 0.28 \mathrm{~nm}$ between $\mathrm{O} 1$ and its two neighbour $\mathrm{O}$ atoms, forming a linear hydrogen bond $\mathrm{O} 0-\mathrm{H} 1-\mathrm{O} 1$ (ESI Fig. S3†). Under the requirement of tetrahedral orientation, we now consider the geometry of the other hydrogen bond O1$\mathrm{H} 2-\mathrm{O} 2$. Since the $\mathrm{H} 1 \mathrm{O} 1 \mathrm{H} 2$ angle is $\sim 109.5^{\circ}$, the $\mathrm{H} 2 \mathrm{O} 1 \mathrm{O} 2$ angle must be $\sim 70.5^{\circ}$. The distance from $\mathrm{O} 2$ to the line $\mathrm{O} 1 \mathrm{H} 2$ is $\sim 0.28 \mathrm{~nm} \times \sin \left(70.5^{\circ}\right)=0.26 \mathrm{~nm}$. This value is much larger than the $\mathrm{O}-\mathrm{H}$ bond length of a water molecule $(\sim 0.096 \mathrm{~nm})$, therefore $\mathrm{H} 2$ cannot make a covalent bond with $\mathrm{O} 2$. In other words, O1 must be the proton-donor. So the $\mathrm{O} 1 \mathrm{H} 2$ length is $\sim 0.096 \mathrm{~nm}$, the $\mathrm{O} 2 \mathrm{H} 2$ length is $\sim 0.263 \mathrm{~nm}$, and the $\mathrm{O} 1 \mathrm{H} 2 \mathrm{O} 2$ angle is $\sim 88^{\circ}$. However, the latter two values do not satisfy the definition of normal hydrogen bonding. Therefore, we can draw a rather general conclusion that linear hydrogen bonds are highly unlikely to exist within a linear row of $\mathrm{O}$ atoms.

In the LD-48MI and MD-HMI, there is no such a linear row, and the angle between two linear hydrogen bonds of an $\mathrm{O}$ atom is around $90^{\circ}$. In the HD-fRMI, however, every nearest neighbor $\mathrm{O}-\mathrm{O}$ pair is in a linear row, so there is no room for a single linear hydrogen bond. In other words, the hydrogen bonding in the HD-fRMI is highly frustrated. This behavior is reflected in the broad and featureless distribution of hydrogen bond angles (Fig. 4c): most $\mathrm{O}-\mathrm{H} \cdots \mathrm{O}$ angles are in the range from $120^{\circ}$ to $150^{\circ}$. In the HD-pRMI, the ridges are still linear rows, but the $\mathrm{O}$ atoms aligned in the direction normal to the ridges form zigzag lines instead of linear rows. Therefore, a half number of hydrogen bonds between nearest-neighboring ridges can be nearly linear (and they are indeed so), leading to notable energy drop (Fig. 9a), while the other half number of hydrogen bonds along the ridges are still frustrated. The latter feature can be seen from the snapshot (Fig. 1d) and the hydrogen-bond angle distribution (Fig. 4c), which shows two peaks around $169^{\circ}$ and 
$138^{\circ}$. This is the reason for the first-order transition between the two rhombic monolayer ices.

Besides the TIP5P model, we have also examined several other three-site and four-site water models (SPC, ${ }^{40} \mathrm{SPC} / \mathrm{E},{ }^{41}$ TIP3P, ${ }^{42}$ TIP4P $\mathrm{P}^{43}$ and TIP4P $/ 2005^{44}$ ). Additional simulations show that all these models give rise to the all-atom planar structure. Our analysis above and results of $a b$ initio calculations demonstrate that the frustrated HD-fRMI structure is more sensible, whereas the all-atom planar structure appears to be unphysical. The key difference between the TIP5P and other models considered may lie in their representation to the electronic structure of $\mathrm{O}$ atoms. The TIP5P model entails two negative charge centers in the directions of the two lone pairs, thus mimicking the $\mathrm{sp}^{3}$ hybridization. Other three-site and foursite models lack the similar feature, and therefore the planar arrangement of four $\mathrm{H}$ atoms around an $\mathrm{O}$ atom results in no energy penalty. This might be a reason why previous simulations on this system did not yield the new structures presented here. One may question why other models can still predict the tetrahedral structure of bulk ice, even though they incur no energy penalty for the flat geometry. An answer is that for the study of bulk ice, the tetrahedral ice is compared with an imaginary structure of all-atom planar layers with no hydrogen bonding between them. Both structures have the same number of near-linear hydrogen bonds (4 per molecule), so the imaginary structure has no advantage in stability. Thus, even without invoking the energy penalty, the tetrahedral ice still wins out because it is more closely packed than the imaginary structure. However, under the highly confined environment such as in the case of the monolayer water, the all-atom planar structure gains a huge advantage in stability over the tetrahedral orientation due to the greater number of near-linear hydrogen bonds (4 versus 0 ). Hence, overlooking the energy penalty in the water model would inevitably lead to the all-atom planar structure. In other words, to capture correct physical chemistry in lowdimensional water and ice, inclusion of the $\mathrm{sp}^{3}$ feature into the water model is fundamentally important.

As shown in Fig. 1c, water molecules in HD-fRMI exhibit two distinct orientations, both tilted to the walls. The orientation of each molecule is different from that of its four neighbors, rendering the $2 \mathrm{D}$ network chessboard-like. Nevertheless, the molecular dipoles with the two orientations result in a net total dipole parallel to the longer diagonal of a rhombus. In HDpRMI, again there are two orientations, while the molecules with the same height have the same orientation. The two orientations are also tilted, and also result in a net total dipole parallel to the longer diagonal of a rhombus. Their polarizations are $18 \mu \mathrm{C} \mathrm{cm}^{-2}$ (HD-fRMI, $D=0.52 \mathrm{~nm}$ ) and $19 \mu \mathrm{C} \mathrm{cm}^{-2}$ (HD-pRMI, $D=0.59 \mathrm{~nm}$ ) and the corresponding $a b$ initio values are $22 \mu \mathrm{C} \mathrm{cm}{ }^{-2}\left(D^{\prime}=0.52 \mathrm{~nm}\right)$ and $23 \mu \mathrm{C} \mathrm{cm}^{-2}\left(D^{\prime}=0.59 \mathrm{~nm}\right)$, respectively (Fig. 8). The ferroelectricities of HD-fRMI and HDpRMI are also confirmed by the strong ferroelectric hysteresis loops (Fig. 7b and c). Also, importantly, the "probably ferroelectric" monolayer ice upon an exposed mica surface observed by Spagnoli et $a .^{31}$ using atomic force microscopy, which appears to exist in a puckered film arrangement, and is consistent with this HD-pRMI.
To study effect of an in-plane electric field on the phase behavior of monolayer water, we perform the same series of simulations for the system subject to a lateral electric field with strength $E_{\mathrm{L}}=0.01,0.1,0.4,1$, and $10 \mathrm{~V} \mathrm{~nm}^{-1}$, respectively. These values are comparable to those experienced by water within the crevices of polar crystals, ${ }^{45}$ from the surfaces of biopolymers ${ }^{46,47}$ or nanoelectrodes. ${ }^{48}$ Fig. $3 \mathrm{~b}-\mathrm{f}$ show the $P_{\mathrm{L}}-D$ phase diagrams with different $E_{\mathrm{L}}$, or $2 \mathrm{D}$ cross-sections of the $3 \mathrm{D}$ $P_{\mathrm{L}}-D-E_{\mathrm{L}}$ phase diagram. The corresponding raw data are given in Fig. S4 as the $\rho-P_{\mathrm{L}}$ curves. $\dagger$

For $E_{\mathrm{L}}=0.01 \mathrm{~V} \mathrm{~nm}^{-1}$, the topology of the phase domains is the same as that in Fig. 3a, but the phase boundaries are relocated. For $E_{\mathrm{L}}=0.1 \mathrm{~V} \mathrm{~nm}^{-1}$, the phase boundaries are moved further away. A qualitative change occurs for $E_{\mathrm{L}}=0.4 \mathrm{~V} \mathrm{~nm}^{-1}$ : the domain of MLiq becomes an "island-like" region, and can no longer separate the two solid-phase regions. For $D \leq 0.557 \mathrm{~nm}$ or $D \geq 0.59 \mathrm{~nm}$, MD-HMI can directly transform into either HDfRMI or HD-pRMI (movies S2 and S3†). The domain of LD-48MI is now fully separated from the MLiq phase by MD-HMI. For $E_{\mathrm{L}}$ $=1 \mathrm{~V} \mathrm{~nm}^{-1}$, both MLiq and LD-48 ML domains disappear, and only the three ferroelectric phases remain. At $E_{\mathrm{L}}=10 \mathrm{~V} \mathrm{~nm}^{-1}$, only the two high-density rhombic phases survive. Obviously, the reason for these behaviors is that the polarization energy under electric field stabilizes the ferroelectric phases.

For given $D$ and $E_{\mathrm{L}}$, increasing $P_{\mathrm{L}}$ favors phases with higher area density $\rho$. The order of $\rho$ (LD-48MI $<$ MD-HMI $<$ MLiq $<$ HD-fRMI $<$ HD-pRMI) is indeed a general sequence of phases in the $P_{\mathrm{L}}$ direction. Moreover, the area density of the monolayer structure is positively correlated with its coordination number $\left(N_{\mathrm{C}}\right)$. The $N_{\mathrm{C}}$ is 3 for LD-48MI and MD-HMI, 4 for HD-fRMI and HD-pRMI, and about 3.5 for MLiq. Hence, the two solids with $N_{\mathrm{C}}$ $=3$ arise at a low pressure region and are next to one another, while the two solids with $N_{\mathrm{C}}=4$ arise at a high pressure region and are next to one another. The MLiq separates the two groups. Because the difference in $\rho$ between HD-fRMI and HD-pRMI is very small, the phase boundary line between them is quite flat.

Among the five 2D phases, three are flat (LD-48MI, MD-HMI, and HD-fRMI), and two are nonplanar (MLiq and HD-pRMI). When increasing $D$ while fixing $P_{\mathrm{L}}$ and $E_{\mathrm{L}}$, each phase would rearrange its structure to lower its free energy, and the nonplanar phases have more flexibility than the planar phases in the structural relaxation. Hence, the domain of HD-pRMI is above that of HD-fRMI. Moreover, since both LD-48MI and MD-HMI are relatively less sensitive to change in $D$, their boundary is nearly vertical. Because MLiq is nonplanar, increasing $D$ would widen its domain in both directions of $P_{\mathrm{L}}$. Therefore, the slopes of the (MD-HMI)-MLiq and MLiq-(HD-fRMI) boundaries are negative and positive, respectively. In the two nonplanar phases, HD-pRMI is more favorable with increasing $D$ due to its longrange ordering, so the slope of the phase boundary is negative. Thus, the MLiq-(HD-fRMI)-(HD-pRMI) triple point is the turning point of the two liquid-solid lines.

\section{Conclusions}

In conclusion, four monolayer ice phases, including two new ones, are predicted based on molecular dynamics simulations. 
Moreover, both new phases (MD-HMI and HD-fRMI) and the HD-pRMI are predicted to be ferroelectric. The latter appears to be relevant to the prediction of "probable ferroelectricity" from experiment. ${ }^{31}$ Stabilities and ferroelectricities of the 2D monolayer ices are supported by the $a b$ initio density-functional theory calculations, as well as by the strong tendency of satisfying the ice rule in a monolayer of water. Both evidences suggest that the existence of $2 \mathrm{D}$ ferroelectric ices is highly plausible and thus warrants future experimental confirmation and more simulations with improved water models (e.g. WAIL water model $^{49}$ ). Finally, we find that an in-plane external electric field can stabilize the ferroelectric phases and eliminate the nonpolarized phases. In contrast, a perpendicular external electric field can melt the monolayer ice. ${ }^{33}$ Thus, an external electric field may be useful to modify phase behavior of confined water.

\section{Acknowledgements}

This work is supported by MOST (2011CB921400), by NSFC (20603032, 20733004, 91021004, 20933006, 21121003, 2123307), by the Fundamental Research Funds for the Central Universities (WK2340000006, WK2060140005, WK2060030012), by CAS (XDB01020300), and by USTCSCC. JB is supported by a grant from UNL Research Council. XCZ is supported by grants from the NSF (CBET-1066947, CHE-1306326), ARL (W911NF1020099), the Nebraska Research Initiative, and a grant from USTC for (1000plan) Qianren-B summer research.

\section{Notes and references}

1 X. Su, L. Lianos, Y. R. Shen and G. A. Somorjai, Phys. Rev. Lett., 1998, 80, 1533.

2 M. J. Iedema, M. J. Dresser, D. L. Doering, J. B. Rowland, W. P. Hess, A. A. Tsekouras and J. P. Cowin, J. Phys. Chem. $B, 1998,102,9203$.

3 K. Koga, G. T. Gao, H. Tanaka and X. C. Zeng, Nature, 2001, 412, 802 .

4 R. J. Mashl, S. Joseph, N. R. Aluru and E. Jakobsson, Nano Lett., 2003, 3, 589.

5 J. Bai, C.-R. Su, R. D. Parra, X. C. Zeng, H. Tanaka, K. Koga and J.-M. Li, J. Chem. Phys., 2003, 118, 3913.

6 J. Bai, J. Wang and X. C. Zeng, Proc. Natl. Acad. Sci. U. S. A., 2006, 103, 19664.

7 D. Takaiwa, I. Hatano, K. Koga and H. Tanaka, Proc. Natl. Acad. Sci. U. S. A., 2008, 105, 39.

8 R. M. Kumar, M. Elango, R. Parthasarathi and V. Subramanian, J. Phys. Chem. A, 2011, 115, 12841.

9 Y. Maniwa, H. Kataura, M. Abe, S. Suzuki, Y. Achiba, H. Kira and K. Matsuda, J. Phys. Soc. Jpn., 2002, 71, 2863.

10 Y. Maniwa, H. Kataura, M. Abe, A. Udaka, S. Suzuki, Y. Achiba, H. Kira, K. Matsuda, H. Kadowaki and Y. Okabe, Chem. Phys. Lett., 2005, 401, 534.

11 S. Ghosh, K. V. Ramanathan and A. K. Sood, Europhys. Lett., 2004, 65, 678.
12 A. I. Kolesnikov, J. M. Zanotti, C. K. Loong, P. Thiyagarajan, A. P. Moravsky, R. O. Loutfy and C. J. Burnham, Phys. Rev. Lett., 2004, 93, 035503.

13 O. Byl, J. C. Liu, Y. Wang, W. L. Yim, J. K. Johnson and J. T. Yates, J. Am. Chem. Soc., 2006, 128, 12090.

14 C. Luo, W. Fa, J. Zhou, J. Dong and X. C. Zeng, Nano Lett., 2008, 8, 2607.

15 H.-X. Zhao, X.-J. Kong, H. Li, Y.-C. Jin, L.-S. Long, X. C. Zeng, R.-B. Huang and L.-S. Zheng, Proc. Natl. Acad. Sci. U. S. A., 2011, 108, 3481.

16 R. Zangi and A. E. Mark, J. Chem. Phys., 2004, 120, 7123.

17 K. Koga, X. C. Zeng and H. Tanaka, Phys. Rev. Lett., 1997, 79, 5262.

18 K. Koga, H. Tanaka and X. C. Zeng, Nature, 2000, 408, 564. 19 R. Zangi and A. E. Mark, J. Chem. Phys., 2003, 119, 1694.

20 S. Han, M. Y. Choi, P. Kumar and H. E. Stanley, Nat. Phys., 2010, 6, 685.

21 J. C. Johnston, N. Kastelowitz and V. Molinero, J. Chem. Phys., 2010, 133, 154516.

22 J. Bai and X. C. Zeng, Proc. Natl. Acad. Sci. U. S. A., 2012, 109, 21240.

23 N. Giovambattista, P. J. Rossky and P. G. Debenedetti, Phys. Rev. Lett., 2009, 102, 050603.

24 G. A. Kimmel, J. Matthiesen, M. Baer, C. J. Mundy, N. G. Petrik, R. S. Smith, Z. Dohnalek and B. D. Kay, J. Am. Chem. Soc., 2009, 131, 12838.

25 J. Yang, S. Meng, L. F. Xu and E. G. Wang, Phys. Rev. Lett., 2004, 92, 146102.

26 J. Bai, C. A. Angell and X. C. Zeng, Proc. Natl. Acad. Sci. U. S. A., 2010, 107, 5718.

27 R. Zangi and A. E. Mark, Phys. Rev. Lett., 2003, 91, 025502.

28 P. Kumar, S. V. Buldyrev, F. W. Starr, N. Giovambattista and H. E. Stanley, Phys. Rev. E: Stat., Nonlinear, Soft Matter Phys., 2005, 72, 051503.

29 K. Koga and H. Tanaka, J. Chem. Phys., 2005, 122, 104711.

30 A. L. Ferguson, N. Giovambattista, P. J. Rossky, A. Z. Panagiotopoulos and P. G. Debenedetti, J. Chem. Phys., 2012, 137, 144501.

31 C. Spagnoli, K. Loos, A. Ulman and M. K. Cowman, J. Am. Chem. Soc., 2003, 125, 7124.

32 M. W. Mahoney and W. L. Jorgensen, J. Chem. Phys., 2000, 112, 8910.

33 H. Qiu and W. Guo, Phys. Rev. Lett., 2013, 110, 195701.

34 B. Hess, C. Kutzner, D. van der Spoel and E. Lindahl, J. Chem. Theory Comput., 2008, 4, 435.

35 S. Nosé and M. L. Klein, Mol. Phys., 1983, 50, 1055; W. G. Hoover, Phys. Rev. A, 1985, 31, 1695.

36 M. Parrinello and A. Rahman, J. Appl. Phys., 1981, 52, 7182.

37 I. C. Yeh and M. L. Berkowitz, J. Chem. Phys., 1999, 111, 3155. 38 T. Kaneko, J. Bai, K. Yasuoka, A. Mitsutake and X. C. Zeng, J. Chem. Theory Comput., 2013, 9, 3299.

39 The CASTEP software code is distributed and maintained by Accelyrs Inc.

40 H. J. C. Berendsen, J. P. M. Postma, W. F. van Gunsteren, and J. Hermans, in Intermolecular Forces, ed. B. Pullman, Reidel, Dordrecht, Holland, 1981, p. 331. 
41 H. J. C. Berendsen, J. R. Grigera and T. P. Aatsma, J. Phys. Chem., 1987, 91, 6269.

42 W. L. Jorgensen, J. Chandrasekhar, J. D. Madura, R. W. Impey and M. L. Klein, J. Chem. Phys., 1983, 79, 926.

43 W. L. Jorgensen and J. D. Madura, Mol. Phys., 1985, 94, 1381. 44 J. L. F. Abascal and C. Vega, J. Chem. Phys., 2005, 123, 234505.

45 M. Gavish, J.-L. Wang, M. Eisenstein, M. Lahav and L. Leiserowitz, Science, 1992, 256, 815.
46 W. Drost-Hansen and J. Lin Singleton, Fundamentals of Medicinal Cell Biology, JAI, Greenwich, CT, 1992, vol. 3a, p. 157.

47 J. A. Fornés, J. Colloid Interface Sci., 2008, 323, 255.

48 D. L. Scovell, T. D. Pinkerton, V. K. Medvedev and E. M. Stuve, Surf. Sci., 2000, 457, 365.

49 Y. Li, J. Li and F. Wang, Proc. Natl. Acad. Sci. U. S. A., 2013, 110, 12209. 